\section{Research-reporting standards fall short}

More than 150 journals and many research-funding charities have endorsed the ARRIVE guidelines for reporting research that uses animal models (C. Kilkenny et al. PLoS Biol. 8, e1000412; 2010), but we find that they are being largely ignored. This could undermine data reproducibility and model credibility, and might obstruct translation into human therapy (S. Landis et al. Nature 490, 187-191; 2012).

For example, of 180 papers on multiple sclerosis listed on PubMed in the past 6 months, we found that only $40 \%$ used appropriate statistics to compare the effects of gene-knockout or treatment. Appropriate statistics were applied in only $4 \%$ of neuroimmunological studies published in the past two years in Nature Publishing Group journals, Science and Cell (details available from D.B. on request). Many journals are therefore failing to ensure that the basics of experimental design and data analysis are respected.

Simply introducing guidelines is not enough. The issue requires greater editorial oversight (perhaps using a tick-box questionnaire at submission), stricter refereeing standards and engagement by learned societies. David Baker, Katie Lidster, Ana Sottomayor Queen Mary, University of London, UK. david.baker@qmul.ac.uk Sandra Amor Free University Amsterdam, the Netherlands.

\section{From pork lard to palm oil and back}

The food industry is rapidly ramping up the production of palm oil, destroying tropical forest at an alarming rate to make way for more oil-palm plantations. Consumers might assume that palm oil is more heart-healthy than animal-based lard. It is not: human metabolism recognizes the chemicals themselves, not their source.

The popularity of butter and lard declined in the 1940s and 1950s, giving way to margarine. This shift was fuelled by the belief that vascular health could be improved by switching from saturated animal fats to unsaturated plant oils. Margarine fell from grace around 1990, when it was discovered that plant-oil solidification produces metabolically harmful trans-fat.

The physical-chemical properties of lard make it ideal for baking, and palm oil is an effective substitute because its chemical composition is almost identical. In lard, the ratios of saturated, monounsaturated and polyunsaturated fatty acids are 43:47:10, whereas in palm oil they are $47: 45: 8$. Neither type contains significant amounts of the more beneficial omega-3 fatty acids.

So we have come full circle. Why discard the pork fat from abattoirs only to replace it with essentially the same thing, but from an ecologically disastrous source?

José Bonner Indiana University, Bloomington, Indiana, USA. bonner@indiana.edu

\section{Strength to strength for mouse models}

Jessica Bolker's emphasis on choosing the right animal model (Nature 491, 31; 2012) should not undermine the validity of the mouse as a model for human disease. Contrary to her implication, mouse researchers do take genetic background and environment into consideration.

Mouse models used to test therapies should reproduce closely the human disease being investigated - including the response to genetic and environmental factors. But this need not apply to models for understanding disease mechanisms: Nobelprizewinning discoveries of embryonic stem cells, the mouse major histocompatibility locus and monoclonal antibodies, for example, all relied on experimental mice and not disease models.

Researchers do not use only inbred strains of mice: they use genetically characterized mouse populations and deploy different genetic backgrounds to identify complex inherited traits. There are large collections of recombinant inbred mouse strains, as well as a public database of more than 3,000 traits (E. J. Chesler Nature Neurosci. 7, 485-486; 2004). The Collaborative Cross reference panel, representing eight inbred strains of lab and wild-derived mice, contains twice the genetic diversity of the entire human population and allows highresolution analysis of phenotypic variations (Genetics 190, 389-401; 2012).

The full potential of the mouse as a model system has yet to be realized. The study of genetic reference populations, along with a library of mutants for every mouse gene, will continue to transform our understanding of human disease.

Klaus Schughart SYSGENET, HZI Braunschweig, University of Veterinary Medicine Hanover, Germany; and University of Tennessee Health Science Center, Memphis, USA.

klaus.schughart@helmholtz-hzi.de Claude Libert SYSGENET, VIBGhent University, Ghent, Belgium. Martien J. Kas SYSGENET, University Medical Center Utrecht, Utrecht, the Netherlands.

\section{Eritrea should choose its own science path}

I find your perspective on the state of science in Eritrea too narrow and founded on questionable assumptions (Nature 491, 8 and Nature 491, 24-26; 2012).

You assume that links with Western institutions are inherently beneficial; that exiled individuals convey an objective picture of the situation inside a closed country; and that disagreements between governments and academic institutions with Western links result from wrongdoing by the government.

Given the complex and difficult recent history of Eritrea, I suggest (without wishing to endorse the regime) that the interests of elite academics, often trained in the West, may not overlap with those of the country's poorer population. And, although international cooperation is part and parcel of academia, recipients of crucial aid are rarely in a position to stand up for their own interests against powerful donors.

Elements of the Eritrean regime want national institutions to survive and eventually flourish without foreign resources, even if that means serious difficulty in the short and medium term. A closed education system, for example, could take a generation to develop the technical expertise and facilities otherwise delivered immediately to a willing aid recipient.

States must decide for themselves on their path to independence. We may not like what they decide, but drawing a line between those who are with us or against us will not benefit the international scientific community, or the vast populations of countries such as Eritrea.

Andrew Isaac Meso Institut de Neurosciences de la Timone, CNRS/Aix-Marseille University, Marseilles, France. andrew.meso@univ-amu.fr

\section{CORRECTION}

A declaration of competing financial interests was omitted from the Correspondence 'NIH chimps: use existing facilities' (Nature 491, 672; 2012). This has been added at http:// go.nature.com/aqezhu. 